\title{
Dynamics of Random Interfaces and Hydrodynamic Limits
}

Fabio Lucio Toninelli (Technical University of Vienna, Austria)

\section{1}

\section{Introduction}

In this article, we will present a panorama of mathematical results and open problems concerning the large-scale properties, both in space and time, of the dynamics of random interfaces. This is a very broad field of research, whose motivation comes from physics [1], and which involves several branches of mathematics, notably probabilities (Markov processes) and analysis (deterministic and stochastic PDEs, calculus of variations), but also combinatorics (random tilings of the plane, Schur processes) and probabilistic algorithms (Markov "Monte Carlo" chains). We will only focus on a few selected aspects.

To introduce the topic, we would first like to take two examples from everyday life which illustrate the type of problems we are talking about. We will then move on to the mathematical modelling of such physical phenomena. As a first example, let us imagine the growth of the snow cover in a garden on a winter's day. Although the trajectory of individual flakes is an essentially random process, if we look at the landscape from a certain distance, we will get the impression that the snow height profile grows in a manner that is regular enough to form a smooth surface. However, if you look closer, you will notice a much more irregular and rough structure, on a larger scale than the typical size of the individual flakes, because of the fact that the flakes do not spread out uniformly on the surface. Note that there are growth phenomena that are of much greater practical importance to study, such as the growth of bacterial colonies in biology or the epitaxial growth of crystals by molecular jet deposition in solid-state physics [1]. Our second example concerns the coexistence of thermodynamic phases. For example, we imagine a container at a temperature of $0^{\circ} \mathrm{C}$ containing ice cubes floating in water. Although at this temperature both ice and water are in a thermodynamically stable state, we can observe a temporal evolution of the shape of the ice cubes (and thus of the water/ice interface). Once again, the evolution appears regular and deterministic on a large scale, and essentially random if we observe the interface under the microscope. Note that this second example is of a very different nature: if the former is a growth phenomenon (the snow cover increases with time), in the latter the water/ice interface is in a state of equilibrium because water and ice are both stable at the particular temperature of $0^{\circ} \mathrm{C}$. This distinction between two very different physical situations will have repercussions on the mathematical models that we will introduce in the following.

The aim of this research area is to understand both the macroscopic (deterministic) evolution on a large scale and the fluctuations around this macroscopic behaviour. Note that the point of view of the mathematician working on these prob- lems is to abstract from the microscopic details of physical or specific biological systems and try to extract "universal" types of behaviour.

Obviously, there is no way to study such physical phenomena on the basis of the fundamental laws governing the movement of water molecules or snow flakes. Following the usual spirit of statistical physics, the idea is rather to introduce strongly simplified mathematical models which, however, retain the essential qualitative aspects of real systems. First of all, the corresponding interface is usually modelled by a realvalued (or, often, integer-valued) height function $h(x)$, where the spatial coordinate $x$ takes its values in a $d$-dimensional network $x \in \mathbb{Z}^{d} . h(x)$ should be interpreted as the vertical coordinate of the interface above point $x$. Note that this type of description is already a huge simplification, not only because physical space is not discrete, but also because any realistic interface will have "overhangs" that prevent us from identifying it with the graph of a function $h$. As to the dimension $d$, the physically most intuitive case is that of $d=2$, in which case the height function describes a two-dimensional interface in the usual three-dimensional space. However, it is interesting to consider the case of any dimension, not only for the sake of mathematical generality, but also because, for example, the one-dimensional case $d=1$ is very rich in physical applications. For example, in the case of a burning sheet of paper, the combustion front is a one-dimensional interface that propagates in a two-dimensional space (the sheet).

A second crucial simplification is to assume that the temporal evolution of the height function $h$ is a stochastic process, and more particularly a time homogeneous Markovian process. The interface will therefore be described by a random function $h(x, t)$, with $t \geq 0$ being a continuous parameter that designates time: the Markov property implies that the probability of transition from a configuration $h_{A}=\left\{h_{A}(x)\right\}_{x \in \mathbb{Z}^{d}}$ at time $t_{A}$ to a configuration $h_{B}=\left\{h_{B}(x)\right\}_{x \in \mathbb{Z}^{d}}$ at time $t_{B}>t_{A}$ depends only on $h_{A}, h_{B}$ and $t_{B}-t_{A}$, and not on the whole history of the system between times 0 and $t_{A}$, nor on the individual values of $t_{A}, t_{B}$. Conceptually, it may seem very risky to replace the deterministic laws of physics by random laws of evolution, but this corresponds exactly to the usual approach of statistical physics to replace the Hamiltonian (deterministic) equations of a set of molecules by a stochastic process. Since the "true" interactions between elementary components (molecules, snow flakes...) of the physical system are essentially local (i.e., of short range) in space, we will choose a Markovian process whose elementary transitions are themselves local: for example, during an elementary transition $h \mapsto h^{\prime}$, the height at a certain point $x$ changes from the value $h(x)$ to the value $h^{\prime}(x)=h(x)+n, n \in \mathbb{Z}$, while the other values remain unchanged. We denote by $c\left(h \rightarrow h^{\prime}\right)>0$ the 


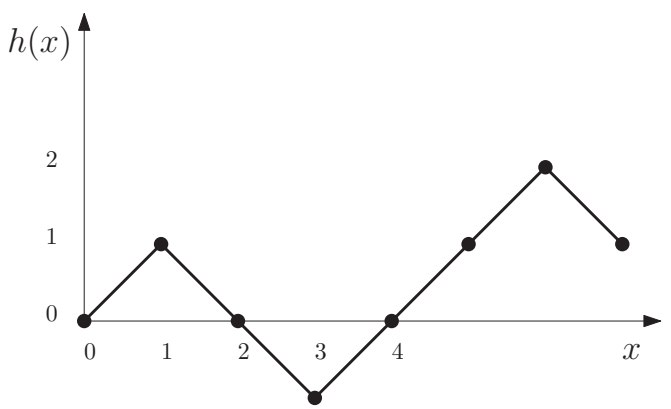

Figure 1. A portion of the height profile of the simple exclusion model

infinitesimal transition rate, i.e., the probability of transition between $h$ et $h^{\prime}$ in an infinitesimal time interval $[0, \delta]$ will be given by $c\left(h \rightarrow h^{\prime}\right) \delta+O\left(\delta^{2}\right)$. To respect the local nature of the physical interactions, it will be assumed that the transition rate depends only on the configuration of $h$ around point $x$, rather than on the function $h$ as a whole.

In order to make this discussion more concrete, we will first describe the most canonical and best studied example of stochastic interface evolution [9]: the one-dimensional simple exclusion process. In this model, the configurations of allowed interfaces $h=\{h(x)\}_{x \in \mathbb{Z}}$ are the following: each height $h(x)$ takes its values in $\mathbb{Z}$ and its gradients $h(x)-h(x-1)$ take only the values +1 or -1 . One can thus visualise such an interface as a one-dimensional zigzag path in two-dimensional space, as in Figure 1.

Now let us describe the associated Markov process. Transitions occur at real random times, and each is an elementary transition of one of two types:

- If $h(x-1)=h(x+1)=h(x)+1$, i.e., if the height has a local minimum in $x$, then we can have the transition $h \rightarrow h^{(x,+)}$, where $h^{(x,+)}(y)=h(y)$ for all $y \neq x$ while $h^{(x,+)}(x)=h(x)+2$ (the local minimum in $x$ becomes a local maximum).

- Inversely, if $h(x-1)=h(x+1)=h(x)-1$ (local maximum in $x$ ) then we can have the transition $h \rightarrow h^{(x,-)}$, where we let $h^{(x,-)}$ be the configuration where the height in $x$ is decreased by 2 , while it is unchanged everywhere else.

We still have to specify the rates associated with these two types of transitions. Given a real number $p \in[0,1]$, we will assign the rate $c\left(h \rightarrow h^{(x,+)}\right):=p$ to transitions that increase the interface, and the rate $c\left(h \rightarrow h^{(x,-)}\right):=1-p$ to those that decrease it. The special case $p=1 / 2$ is known as the Symmetric Simple Exclusion Process (SSEP), the case $p \neq$ $1 / 2$ is the Asymmetric Simple Exclusion Process (ASEP) and the special case $p \in\{0,1\}$, where only transitions of one of the two types are allowed, is known as Totally Asymmetric Simple Exclusion (TASEP).

An equivalent description of the simple exclusion process is as follows: each $x$ has an independent random clock which rings at random exponential time intervals of mean 1 . If the clock in $x$ rings at time $t$, a coin is tossed which gives $\wedge$ with probability $p$ and $\vee$ with probability $1-p$. If we obtain $\wedge$ and the height function at time $t$ has a local minimum in $x$, we then perform the transition $h \mapsto h^{(x,+)}$, otherwise we do nothing; analogously, if we get $\mathrm{V}$ and the height function at time $t$ has a local maximum in $x$, we then perform the transition $h \mapsto$ $h^{(x,-)}$, otherwise we do nothing. Note that, since the clocks at two points $x \neq x^{\prime}$ are independent, we will never (except with zero probability) have two clocks ringing at the same instant of time, and thus each transition changes the height $h$ at a single point $x$.

As we said at the very beginning of this note, we are interested in the large-scale behaviour of interface dynamics. There are several ways to understand the expression "largescale behaviour". To understand this point, let us forget for a moment about interface dynamics and think about a much simpler probabilistic question: the asymptotic behaviour for large $N$ of the sum

$$
S_{N}=\sum_{i=1}^{N} X_{i}
$$

of $N$ independent and equally distributed random variables $X_{i}$ which, for simplicity, are assumed to have a finite variance

$$
\sigma^{2}:=\mathbb{E}\left(X_{1}^{2}\right)-\left(\mathbb{E} X_{1}\right)^{2}<\infty,
$$

where $\mathbb{E}(f)$ denotes the mean of a random variable $f$. The first question is, what is the asymptotic behaviour of $(1 / N) S_{N}$, and the well-known answer is the law of large numbers

$$
\lim _{N \rightarrow \infty} \frac{S_{N}}{N}=\mathbb{E}\left(X_{1}\right)
$$

where the convergence of the random variable $S_{N} / N$ towards the deterministic quantity $\mathbb{E}\left(X_{1}\right)$ is valid with probability 1 . Next, there is the question of what the fluctuations are of $S_{N} / N$ around its deterministic limit: we will obtain the central limit theorem

$$
\sqrt{N}\left(\frac{S_{N}}{N}-\mathbb{E}\left(X_{1}\right)\right) \stackrel{N \rightarrow \infty}{\Rightarrow} \mathcal{N}\left(0, \sigma^{2}\right)
$$

where $\mathcal{N}\left(0, \sigma^{2}\right)$ is a random normal (i.e., Gaussian) centered variable of variance $\sigma^{2}$ and " $\Rightarrow$ " means that the law of the random variable on the left tends towards the law of the random variable on the right. If the central limit theorem describes typical or "normal" fluctuations of $S_{N} / N$ at scale $1 / \sqrt{N}$, we can also be interested in atypical deviations (say, deviations of order 1). We will then have statements of the "large deviations" type:

$$
-\lim _{N \rightarrow \infty} \frac{1}{N} \log \mathbb{P}\left(\left|\frac{S_{N}}{N}-\mathbb{E}\left(X_{1}\right)\right| \geq \delta\right)=I(\delta)
$$

for a certain function $I(\cdot)$ which depends on the law of variables $X_{i}$. Here, $\mathbb{P}(A)$ denotes the probability of an event $A$. Although the evolution of a random interface is something much more complicated than a sum of independent variables, these three types of questions (law of large numbers, central limit theorem, large deviations) are also very natural in this context.

In this discussion, for interface dynamics, we will concentrate mainly on the first two questions ("law of large numbers" and "fluctuations"). In the context of interface dynamics, but more generally of out-of-equilibrium statistical physics, we will rather speak of "hydrodynamic limit" than "law of large numbers". 1 The question can be asked as follows. Suppose

1 The terminology of "hydrodynamic limit" comes from fluid dynamics: if the motion of molecules is governed at the microscopic level by Hamiltonian equations with chaotic and essentially random behaviour, at the laboratory scale we observe the deterministic evolution of a finite number of macroscopic parameters (pressure, density, ...), described by partial differential equations. 
that the initial condition of the dynamics, i.e., the configuration $h(\cdot, 0)=\{h(x, 0)\}_{x \in \mathbb{Z}^{d}}$ of height at time $t=0$, approximates a limit height profile $\phi_{0}: \mathbb{R}^{d} \mapsto \mathbb{R}$. More precisely, let us assume that the initial profile $h(\cdot, 0)$ satisfies, for all $u \in \mathbb{R}^{d}$,

$$
\lim _{\epsilon \rightarrow 0} \epsilon h\left(\left\lfloor\epsilon^{-1} u\right\rfloor, 0\right)=\phi_{0}(u) .
$$

For example, for the simple exclusion process, given a function $\phi_{0}$ of Lipschitz constant inferior to 1 , we could choose $h(x, 0)$ as being the nearest integer to

$$
\frac{1}{\epsilon} \phi_{0}(\epsilon x)
$$

and having the same parity as $x$ (the latter condition ensures that $h(x, 0)-h(x-1,0) \in\{-1,+1\})$. We say that a dynamic admits a hydrodynamic limit if there is a deterministic function $\phi=\phi(u, t), t \geq 0, u \in \mathbb{R}^{d}$ and an exponent $\gamma \geq 0$ such that

$$
\lim _{\epsilon \rightarrow 0} \epsilon h\left(\left\lfloor\epsilon^{-1} u\right\rfloor, \epsilon^{-\gamma} t\right)=\phi(u, t) .
$$

Here, the convergence has to be interpreted as a convergence in probability: for all $\delta>0$,

$$
\lim _{\epsilon \rightarrow 0} \mathbb{P}\left(\left|\epsilon h\left(\left\lfloor\epsilon^{-1} u\right\rfloor, \epsilon^{-\gamma} t\right)-\phi(u, t)\right| \geq \delta\right)=0 .
$$

As can easily be imagined, there will only be one choice for the exponent $\gamma$ that allows us to find a well-defined and nottrivial hydrodynamic limit. For example, if $\gamma$ is too small (e.g., $\gamma=0)$ we have $\phi(x, t)=\phi(x, 0)$ for all $t$. In all the examples discussed in this note, this exponent is either $\gamma=1$ or $\gamma=2$. In the first case, we will say that we have a hydrodynamic limit in the Eulerian (or hyperbolic) scale, while in the second case we will speak of a diffusive scale. Basically, the interface growth models (such as the example of snowfall) correspond to the Eulerian scale, while the interface dynamics models describing the motion of boundaries between coexisting thermodynamic phases (such as the example of the water/ice interface at $0^{\circ} \mathrm{C}$ ) correspond to the diffusive scale.

Let us illustrate this fact by returning to the case of simple exclusion. Let us first take the case $p=1$ (or more generally $p \neq 1 / 2$ ). In this case, the growth of the interface is intrinsically irreversible: only transitions where the height increases are allowed. If we exclude the pathological case where the macroscopic slope $\rho$ of the interface is \pm 1 , we have a positive density $r=(1+\rho) / 2$ of points $x$ with $h(x)-h(x-1)=1$, and a positive density $r=(1-\rho) / 2$ of points $x$ with $h(x)-h(x-1)=-1$. There will thus also be a positive density of points $x$ where $h(\cdot)$ has a local maximum (resp. a local minimum). Since in a time of order 1 any local minimum has a probability of order 1 of becoming a local maximum, it is intuitive that after a time of order $t$, on average the height function increases by an amount of order $t$. One must therefore choose $\gamma=1$ to hope to obtain a nontrivial hydrodynamic limit. The situation is very different for the symmetric exclusion where $p=1 / 2$ : in this case, since the positive and negative transitions tend to compensate each other (just as ice cubes have no natural tendency to grow or melt at $0^{\circ} \mathrm{C}$ ), one must look at much longer time scales to see a non-trivial evolution. The fact that $\gamma=2$ is the right choice requires a little more thought and we refer the reader to the discussion in Section 2.

Since we mentioned the word "irreversible", the following observation should be made. Suppose that the height profile $\{h(x)\}_{x \in \mathbb{Z}}$ at time zero is random and in particular that each gradient $h(x)-h(x-1)$ is independent and takes on the value +1 with probability $r$ and the value -1 with probability $1-r$, for a certain $r \in(0,1)$. It is easy to verify that, both for the simple symmetric and for the asymmetric exclusion, such a distribution is invariant for the dynamics: the law of gradients $h(x, t)-h(x-1, t)$ will be exactly the same at time $t>0$. It is thus a stationary state (or invariant measure: we will use these two terms as synonyms). What distinguishes the symmetric case $p=1 / 2$ from the asymmetric case is therefore not the invariant measure, but the reversibility. Let us suppose that at initial time (and thus for all times) the gradients are distributed according to the stationary measure, and compare the law of the gradient process $\{h(x, t)-h(x-1, t)\}_{x \in \mathbb{Z}, t \geq 0}$ and that of the process after time reversal, $\{h(x,-t)-h(x-1,-t)\}_{x \in \mathbb{Z}, t \geq 0}$. It turns out that these two processes are governed by the same law (i.e., the process is reversible) if and only if $p=1 / 2$.

It's natural to ask what kind of evolution will follow the "hydrodynamic" profile $\phi(u, t)$. If, as for the simple exclusion, we started from a Markovian evolution whose transition rates depend only on the local gradients of the configuration $h$, we can expect that $\partial_{t} \phi(u, t)$ depends only on the spatial derivatives of $\phi(u, t)$ at point $u$. In other words, we expect that $\phi$ follows a PDE. More precisely, it turns out that:

- in the case $\gamma=2$ the hydrodynamic PDE will be of the parabolic type:

$$
\partial_{t} \phi(u, t)=\mu(\nabla \phi(u, t)) \sum_{i, j=1}^{d} \sigma_{i, j}(\nabla \phi(u, t)) \partial_{u_{i} u_{j}}^{2} \phi(u, t)
$$

with $\mu(\nabla \phi)>0$ a function which is named "mobility" and $\left(\sigma_{i, j}\right)_{i, j=1, \ldots, d}$ a symmetric and positive definite matrix whose elements are the second derivatives of the surface tension $\sigma$ of the model. For the reader willing to understand the physical meaning of $\mu$ and $\sigma$, we refer to the very instructive discussion of [14];

- in the case of growth models where $\gamma=1$, the PDE will be of the "Hamilton-Jacobi" type

$$
\partial_{t} \phi(u, t)=v(\nabla \phi(u, t)) .
$$

The function $v(\cdot)$, generally non-linear, describes the interface growth rate as a function of the local slope $\nabla \phi$.

Note that, as a consequence of (2), the hydrodynamic PDE must be invariant by reparameterisation $\phi(u, t)$ $\mapsto c \phi\left(c^{-1} u, c^{-\gamma} t\right)$ for all $c>0$. Indeed, the two equations (3) and (4) satisfy this property.

Let us return to our analogy with the sum of independent random variables: after the law of large numbers, it is the turn of fluctuations. For diffusive models such as the simple symmetric exclusion or its analogues in dimension $d \geq 1$, let us define the "height fluctuation field" as

$$
\hat{h}^{(\epsilon)}(u, t):=\epsilon^{1-d / 2}\left\{h\left(\left\lfloor\epsilon^{-1} u\right\rfloor, \epsilon^{-2} t\right)-\mathbb{E} h\left(\left\lfloor\epsilon^{-1} u\right\rfloor, \epsilon^{-2} t\right)\right\} .
$$

Although $\hat{h}^{(\epsilon)}$ is not a sum of independent variables, its randomness results from the cumulative effect of many random events (the transitions of the dynamics in a time interval of the order $\epsilon^{-2}$ ); it is therefore natural to expect a central limit theorem, i.e., that $\hat{h}^{(\epsilon)}(u, t)$ tends towards a Gaussian process for $\epsilon \rightarrow 0$. More precisely, suppose for simplicity that the initial condition is such that the profile $\phi_{0}$ (cf. (1)) is affine: $\phi_{0}(u)=\rho \cdot u, \rho \in \mathbb{R}^{d}$, in such a way that the solution of the 
hydrodynamic equation (3) is simply $\phi(u, t)=\phi_{0}(u)=\rho \cdot u$. Under fairly general assumptions, one would expect $[13,14]$ that the fluctuation field (5) converges, in the sense of distributions, towards the solution $\hat{\phi}$ of the following linear SPDE (stochastic PDE):

$$
\begin{aligned}
\partial_{t} \hat{\phi}(u, t)=\mu(\nabla \phi) \sum_{i, j=1}^{d} \sigma_{i, j}(\nabla \phi) \partial_{u_{i} u_{j}}^{2} & \hat{\phi}(u, t) \\
& +\sqrt{2 \mu(\nabla \phi)} \dot{W}(u, t) .
\end{aligned}
$$

This equation requires explanations. First of all, $\dot{W}(u, t)$ denotes the "space-time white noise", i.e., a Gaussian random function (more precisely, a random distribution) indexed by the space $u$ and the time $t$, of mean zero $(\mathbb{E} \dot{W}(u, t)=0)$ and covariance

$$
\mathbb{E} \dot{W}(u, t) \dot{W}(v, s)=\delta(t-s) \delta(u-v) .
$$

Note, furthermore, that the equation is linear in $\hat{\phi}$, since $\nabla \phi=\rho$ is a constant. For this reason, the solution $\hat{\phi}(u, t)$ is a Gaussian process. The origin of the equation (6) is quite easy to understand intuitively: the first term, proportional to the second derivatives, is obtained by writing the height function $h$ as its hydrodynamic limit $\phi(u, t)$ plus fluctuations $\hat{h}$, and by linearising the hydrodynamic PDE (3) around $\phi(\cdot, \cdot)$. As for the noise, to understand the choice of the factor $\sqrt{\ldots}$, which guarantees the correct stationary measure of the process (6) when $t \rightarrow \infty$, we refer to the discussion in [13, Sec. II.3.5] done in a similar context.

The reader might expect that for growth models (such as TASEP, for example), the fluctuation field also tends towards the solution of a certain SPDE. However, this is not generally the case, and indeed the mathematical (and even heuristic) understanding of this question is still very incomplete. We are going to explain a little more about this in Section 3.

The remainder of this paper is organised as follows. In Section 2 we will discuss reversible interface dynamics, which correspond to $\gamma=2$ and generalise our elementary example of symmetric simple exclusion. In Section 3 we discuss growth models. In particular, we will focus on the case of $d=2$, where there is a non-trivial relationship between the interface fluctuations and the convexity properties of the function $v: \mathbb{R}^{d} \mapsto \mathbb{R}$ that appears in the PDE (4).

To conclude this introduction, it is important to make two remarks:

- As is often the case in statistical physics, on the basis of physical intuition, some phenomena are expected to be qualitatively "universal", i.e., independent of the models' details. In our context, this is the case, for example, for the convergence towards hydrodynamic PDEs of the type (3)(4), or the convergence of fluctuations towards the SPDE (6) in the diffusive case. However, it is only in very specific cases that mathematical proofs can be obtained. For example, with respect to the convergence of the fluctuation field to the solution of (6) for interface dynamics in dimension $d>1$, the reference [7] is essentially the only known result.

- Most of the known mathematical results in this area are specific to the case of the spatial dimension $d=1$. The results in dimensions $d \geq 2$ are much rarer and we will mention some of the most recent ones.

\section{Reversible interface dynamics}

Until quite recently, the only example of reversible interface dynamics in dimension $d>1$ for which a hydrodynamic limit of the type (2)-(3) was mathematically proven was the Ginzburg-Landau type gradient model with symmetric and convex potential [6]. This is a model of a very different nature from simple exclusion, since the height variables take on continuous real values and their evolution does not proceed by discrete jumps, but follows a "Langevin" type dynamic. This means that each height $h(x)$ is subject to independent Brownian noises, with a drift that depends on the differences $h(x)-h(y)$ for all $y$ neighbouring $x$ and that acts as a restoring force that tends to flatten the interface by penalising large gradients. The limit PDE obtained is of a very special type, since the mobility coefficient $\mu(\cdot)$ in (3) is constant. It is natural to wonder if there are other examples of non-trivial dynamics in dimension $d>1$ where the mathematical proof of (2)-(3) can be obtained, and in particular if we can find examples - if possible somewhat closer in spirit to the simple exclusion with non-constant mobility $\mu(\cdot)$.

To do this, take for a moment our example of simple symmetric exclusion and suppose that the interface is defined not on all $\mathbb{Z}$ but on a one-dimensional torus of size $1 / \epsilon$, which is supposed to be an even integer. In other words, the height function is $\{h(x)\}_{x=0, \ldots, 1 / \epsilon}$ with the periodicity constraint $h(0) \equiv h\left(\epsilon^{-1}\right)$ and, as before, $h(x)-h(x-1)= \pm 1$. Given the height function $h(\cdot, t)$ at a time $t$, we calculate its average value at time $t+\delta$, with $\delta$ being infinitesimal. If the interface has a local minimum (respectively a local maximum) at point $x$, i.e., if $\Delta h(x, t)=+2$ (resp. $=-2)$, then the height in $x$ changes by +2 (resp. by -2 ) with probability $p \times\left(\delta+O\left(\delta^{2}\right)\right)=\delta / 2+O\left(\delta^{2}\right)$. It can be easily deduced that

$$
\left.\frac{d}{d s} \mathbb{E}[h(x, t+s) \mid h(\cdot, t)]\right|_{s=0^{+}}=\frac{1}{2} \Delta h(x, t),
$$

where $\mathbb{E}[\ldots \mid h(\cdot, t)]$ denotes the expectation conditioned to the height configuration at time $t$. Thus, we have that the time derivative of the height is proportional to the Laplacian of the height itself. Now, if we define $\tilde{h}(u, t):=\epsilon h\left(\epsilon^{-1} u, t\right)$ with $u=$ $0, \epsilon, 2 \epsilon \ldots, 1$, the same computation leads to

$$
\left.\frac{d}{d s} \mathbb{E}[\tilde{h}(u, t+s) \mid \tilde{h}(\cdot, t)]\right|_{s=0^{+}}=\frac{\epsilon^{2}}{2} \Delta \tilde{h}(u, t),
$$

From there, one can be immediately convinced that the right time scale is diffusive $(\gamma=2)$ and that the hydrodynamic equation (3) of the simple symmetric exclusion will simply be the heat equation:

$$
\partial_{t} \phi(u, t)=\frac{1}{2} \partial_{u}^{2} \phi(u, t) .
$$

Indeed, it is not difficult to show convergence in the form (2), see e.g. [9, Ch. 4]. The example of the simple symmetric exclusion is instructive but very special: the time derivative of the height is given by a linear operator (the Laplacian) applied to the height itself, as shown in (7).

This type of miracle does not usually happen. Let us try to generalise the simple symmetric exclusion model to the dimension $d=2$. If the height profile of the one-dimensional case is a zigzag path in the plane, the most natural twodimensional analogue is a discrete interface like in the Figure 2. 


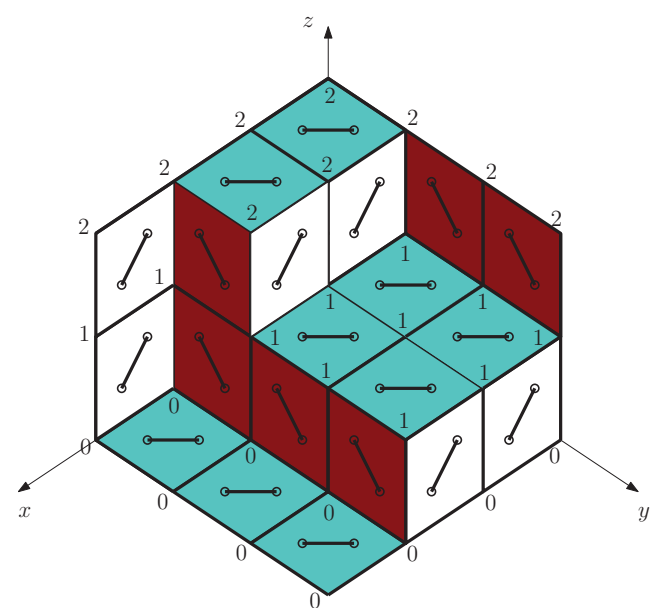

Figure 2. A monotone two-dimensional interface: the height function is (weakly) decreasing in $x$ and $y$ direction. Each level line is a zigzag path as in figure 1. The different contour lines do not intersect.

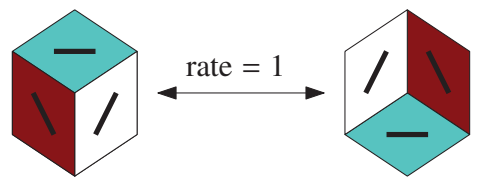

Figure 3. The elementary transitions of the dynamics correspond to adding or removing a unit cube.

There are several equivalent ways of interpreting this image. It can be seen as a tiling of the plane with three types of rhombus-shaped tiles, or as a monotone stack of cubes ("monotone" in the sense that the height of a column of cubes whose base coordinates are $(x, y)$ is decreasing with respect to $x$ and $y$; it is therefore a plane partition), or as a perfect matching of the hexagonal graph (the small segments designate the pairs of vertices that are matched). Note that any section of this interface through a plane perpendicular to one of the three axes gives a zigzag path, thus a height profile of the one-dimensional simple exclusion. However, these onedimensional sections satisfy non-trivial non-intersection constraints and are therefore not independent.

A Markovian dynamic that naturally generalises the onedimensional symmetric simple exclusion to the two-dimensional case is the one where the possible elementary transitions correspond to adding or removing an elementary cube, with transition rate 1: see Figure 3. Unfortunately, things are not nearly as easy as they are for the one-dimensional simple exclusion. Let us try to repeat the same calculation as (7). For that, for any vertex $x$ of a rhombus, we note $h(x) \in \mathbb{Z}$ the height of the vertex of the corresponding cube with respect to the horizontal plane, as in Figure 2. We get

$$
\left.\frac{d}{d s} \mathbb{E}[h(x, t+s) \mid h(\cdot, t)]\right|_{s=0^{+}}=A_{x}-B_{x},
$$

where $A_{x}$ (resp. $B_{x}$ ) is 1 if the configuration around $x$ at time $t$ is as in the drawing on the right (resp. left) side of Figure 3, and 0 otherwise. There are two important differences as compared to Eq. (7): firstly, we have not obtained a closed equation for the height function; secondly, the result is not given by a discrete second-order operator applied to the height function, so it is not obvious a priori that the hydrodynamic equation (if it exists) is of the parabolic type.

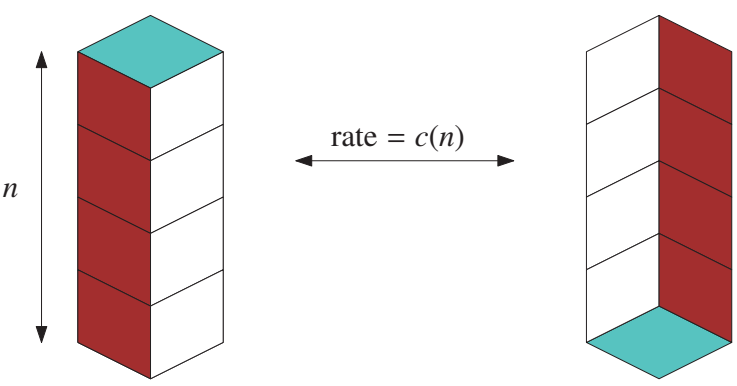

Figure 4

Indeed, it is an open problem (probably very difficult) to show that this dynamic admits a hydrodynamic limit: even from the heuristic point of view, we cannot guess an exact expression for the mobility $\mu$ which should appear in (3). We can be less ambitious and hope to show at least that the right time scale is diffusive. More precisely, consider the same tiling dynamics not in the whole plane, but in a two-dimensional domain of size $1 / \epsilon$ (e.g., a two-dimensional torus). The only invariant (and reversible) probability measure of this dynamic is the uniform measure, which gives equal weight to any allowed tiling of the domain. Given a small constant $\delta$ (say $\delta=1 / 100$ ), we define the mixing time $T_{m i x}$ of the dynamics as the smallest value of $t$ such that, for any initial condition, the law of the process at time $t$ is at a distance (in total variation) at most $\delta$ from the invariant measure. It is reasonable to ask how $T_{m i x}$ grows with the size $1 / \epsilon$ of the system. If we interpret Markov dynamics as a probabilistic algorithm (of the "Markov Chain Monte Carlo" type) that takes a tiling $\eta_{0}$ at step zero and outputs the tiling $\eta_{T}$ at time $T$, then having an upper bound $T_{m i x} \leq N$ implies that the algorithm produces, after a simulation time $N$, an almost perfectly uniform tiling (up to some $\delta$ ). This kind of question about the speed of convergence of probabilistic algorithms is very much studied in theoretical computer science. Coming back to our twodimensional interface dynamics, an important conjecture in this domain is that $T_{m i x}$ is of order $\epsilon^{-2+o(1)}$. Under certain assumptions about the shape of the domain, this conjecture has been proven recently $[4,10]$. The proof uses large-scale properties of uniform tilings of large planar domains, including the fact that their height fluctuations are only logarithmic with respect to the size of the domain. However, the issue is still far from being solved:

Open problem. For the dynamics restricted to a hexagonal domain of side $1 / \epsilon$ (like in Figure 2 where $1 / \epsilon=3$ ) show that $T_{\text {mix }}=O\left(\epsilon^{-2+o(1)}\right)$ for $\epsilon \rightarrow 0$. At present, the best rigorous upper bound is of order $\epsilon^{-4+o(1)}$.

Let us return to the problem of the hydrodynamic limit for the dynamics of monotone two-dimensional interfaces. Since this question is too difficult for the dynamics whose elementary updates are those of the Figure 3, we may ask whether a change in the transition rules for the Markov process can simplify things. For example, one could decide to allow more general transitions where $n$ cubes are added/removed at a time, with a transition rate $c(n)$. See Figure 4. In the recent work [12], we showed, with some heuristic approximations, that if we choose $c(n)$ as being inversely proportional to $n$, the time derivative of the average height $h(x)$ is given by a discrete second-order operator acting on $h(\cdot)$. This suggested the 
possibility of obtaining a hydrodynamic limit in the diffusive time scale. In a later paper [11], we indeed succeeded in rigorously proving that for this dynamic there is a convergence as in (2), where $\phi$ satisfies a non-linear parabolic PDE of the type (3). The PDE is completely explicit and, in particular, the mobility coefficient $\mu(\cdot)$ is non-linear. A study of the hydrodynamic PDE and the specific form of the mobility $\mu(\cdot)$ shows that the equation has remarkable analytical properties: in particular it contracts both the $L^{1}$ and $L^{2}$ distances between its solutions (which is also true for the heat equation, but not for any equation of the form (3)). We refer the reader to [12] for a discussion of the relationship between these analytical properties and the fact that, for our particular choice of transition rate $c(n) \propto 1 / n$, the stochastic process

$$
t \mapsto V(t):=\sum_{x}\left(h^{(1)}(x, t)-h^{(2)}(x, t)\right)
$$

has the property of being decreasing on average (more precisely, it is a supermartingale). Here, $h^{(j)}(\cdot, t), j=1,2$ denote two height profiles who follow the Markovian dynamics with two different initial conditions $h^{(j)}(\cdot, 0)$, for which we assume that $h^{(1)}(\cdot, 0) \geq h^{(2)}(\cdot, 0)$, while $V(t)$ denotes their volume difference.

\section{Models of interface growth}

The mathematical study of one-dimensional interface growth models, such as TASEP and its generalisations, has been extremely active for several years. A remarkable aspect is the emergence of universal non-Gaussian distributions for height fluctuations $h(x, t)-h(x, 0)$ in the long time limit. Moreover, the asymptotic evolution of the fluctuation field is not governed by a SPDE, but a stochastic process ("KPZ fixed point") which is not yet fully understood. We are not going to develop this topic, nor its links with the one-dimensional Kardar-Parisi-Zhang (KPZ) equation (a highly singular nonlinear SPDE), or with the eigenvalue statistics of large random matrices, among others, as these topics have already been the subject of several recent review articles, e.g., [5].

Instead, we will talk about growth models in dimension $d=2$, a much less explored and known field, where new phenomena emerge. We will start with a few generalities. Given a Markovian growth model, such as TASEP for example, there are very natural quantities associated with it. (We stress, however, that in general it is a major mathematical challenge to prove that these quantities are well defined.) Suppose that the initial condition of the dynamics is a deterministic affine profile $h(x)$ of slope $\rho \in \mathbb{R}^{d}$, i.e., $h(x)=x \cdot \rho$ (one may have to take the integer part if the height function is discrete). It is intuitive (but it may be difficult to show in concrete examples!) that:

- the law of gradients $\left\{h(x, t)-h\left(x^{\prime}, t\right)\right\}$, with $x \in \mathbb{Z}^{d}$ and $x^{\prime}$ which takes its values among the $2 d$ neighbors of $x$, tends for $t \rightarrow \infty$ towards a limit law $\pi_{\rho}$, stationary and invariant by translations;

- there is an asymptotic growth rate $v=v(\rho)$, i.e.,

$$
\lim _{t \rightarrow \infty} \frac{1}{t}(h(x, t)-h(x, 0))=v(\rho)
$$

and the function $v(\cdot)$ is the same as in the hydrodynamic PDE (4);
- the typical fluctuations of $h(x, t)-h(x, 0)$ (measured for example by their standard deviation) behave for large $t$ like $t^{\beta} ; \beta \in \mathbb{R}$ is called the growth exponent;

- the typical fluctuations of $h(x, t)-h(y, t)$ for $t \rightarrow \infty$ and $|x-y|$ large behave like $|x-y|^{\alpha}$, with $\alpha \in \mathbb{R}$ being called the roughness exponent because the larger the exponent, the greater the fluctuations in the asymptotic height profile, as compared with an affine profile.

In the particular case of the dimension $d=2$ we have the following conjecture, which links the convexity properties of $v(\cdot)$ to the exponents $\alpha, \beta$ :

Conjecture 1. Let $\lambda_{1}, \lambda_{2}$ be the eigenvalues of the Hessian matrix $D^{2} v$ of $v(\cdot)$. If the product $\lambda_{1} \lambda_{2}$ is negative or null (so if $\left.\operatorname{det}\left(D^{2} v\right) \leq 0\right)$, then $\alpha=\beta=0$ and the growth of fluctuations for $t$ or $|x-y|$ that tend to infinity is only logarithmic. If on the other hand $\lambda_{1} \lambda_{2}>0$, then $\alpha$ and $\beta$ are strictly positive and universal exponents (i.e., independent of the particular values of $\lambda_{i}$ ).

Note that $\lambda_{i}$ are functions of the slope $\rho$ and therefore the sign of $\operatorname{det}\left(D^{2} v\right)$ (and thus the values of the critical exponents) could in principle depend on $\rho$.

Conjecture 1 is a fairly typical universality statement in statistical physics: it is a fairly common fact that the microscopic models that describe a certain macroscopic phenomenon (here, the growth phenomenon) are divided into a small number (here, two) of "universality classes". The models in the same class are characterised by the same "critical exponents" (here, $\alpha$ and $\beta$ ) and the class a model belongs to is determined by certain qualitative symmetries (here, the sign of $\lambda_{1} \lambda_{2}$, thus the convexity properties of $\left.v(\cdot)\right)$. A small note on nomenclature: we will say that

- a growth model for which $\lambda_{1} \lambda_{2}>0$ belongs to the $K P Z$ universality class

- a growth model for which $\lambda_{1} \lambda_{2} \leq 0$ belongs to the Anisotropic KPZ (or AKPZ) universality class.

The above conjecture, which may seem arbitrary at first glance, is based on non-rigorous calculations by physicists [1] and is convincingly confirmed by numerical simulations of several growth models; we refer the reader to [15] for discussions and references. In the remainder of this section, our goal is to give an example of models for each of the two universality classes and to discuss one or two recent mathematical results that shed some light on the conjecture.

To make the discussion more concrete, we discuss two specific two-dimensional growth models. In order to make the link with the discussion of the previous section easier, suppose that the height profiles allowed for our interface are again monotone discrete functions corresponding to rhombus tilings of the plane, like in the Figure 2. In Section 2 our most natural candidate for a reversible dynamic for this interface was the Markovian process whose elementary transitions are those of the Figure 3, with symmetric rates. On the other hand, the natural candidate for a growth process is to allow only one of these two types of transition, for example one that adds an elementary cube, see Figure 5. This growth model is attractive from several points of view:

- it is very easy to be numerically defined, visualised and simulated;

- it is not difficult to show, by a super-additivity argument, 


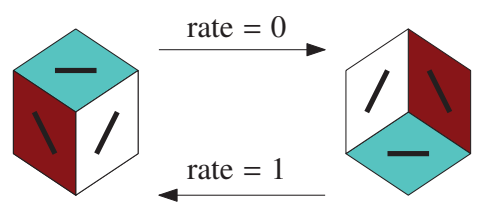

Figure 5. If we allow only those transitions that add a cube, we obtain a growth model that belongs to the KPZ universality class.

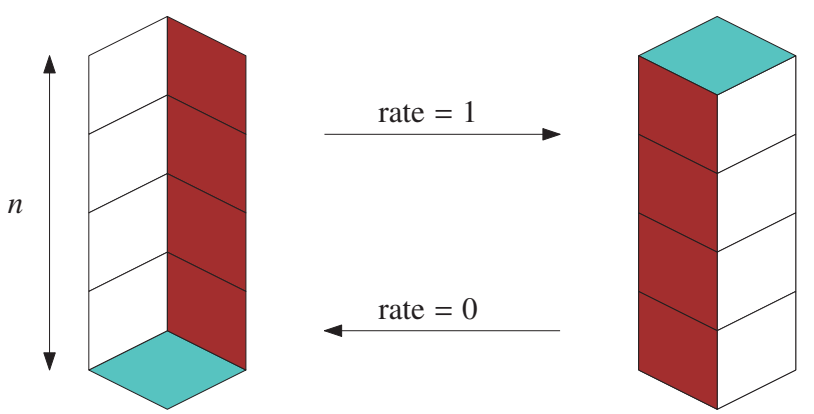

Figure 6. In the growth model studied in [2] we allow transitions that add $n$ cubes at a time and the transition rate does not depend on $n$.

that the limiting velocity $v(\cdot)$ exists, is convex, and that the height profile satisfies a hydrodynamic limit as in (2) at Eulerian scale $\gamma=1$, where the limiting PDE is given by (4). Two remarks: the convexity of $v(\cdot)$ implies that its Hessian matrix will be positive definite and (assuming that its eigenvalues are strictly positive, which has not been rigorously shown because $v(\cdot)$ is not explicitly known) we have a candidate for the KPZ universality class. Secondly, it is well known that non-linear Hamilton-Jacobi equations of the type (4) develop singularities in finite time; however, when $v(\cdot)$ is convex, a natural way to obtain a (weak) solution defined globally in time is through the Hopf-Lax variational formula. Indeed, the super-additivity argument mentioned above gives the convergence of the height profile precisely towards this solution! Unfortunately, the mathematical study of this growth process stops here: we have no idea of how the translation invariant, stationary states $\pi_{\rho}$ look like, there are no mathematical results on the critical exponents $\alpha, \beta$ (although they are known with great precision on the basis of numerical simulations!), nor an explicit expression for $v(\cdot)$. Indeed, we do not know any mathematical result on stationary states or on critical exponents for any two-dimensional growth model of the KPZ universality class.

As in the case of reversible dynamics, one may wonder whether things change qualitatively if we modify the transition rules of the Markov process a little. One possibility is generalising the growth process by allowing one, during each transition, to add $n \geq 1$ cubes at a time, as in the Figure 6 . Note that the transition rate is chosen here independently of the value of $n$. This growth process was introduced in [2] and, quite surprisingly, it turns out that (see [15] for precise bibliographic references):

(i) the speed $v(\cdot)$ can be calculated explicitly and its Hessian has two eigenvalues of opposite sign;

(ii) the critical exponents $\alpha$ and $\beta$ are zero;

(iii) the stationary states $\pi_{\rho}$ can be explicitly determined;

(iv) under certain restrictions on the initial height profile, one can show convergence (in the sense of (2), with $\gamma=1)$ to the viscosity solution of the PDE (4). (The viscosity solution is a weak solution which simply reduces to the Hopf-Lax solution in the case of where $v(\cdot)$ is convex.)

This growth model is therefore a representative of the Anisotropic KPZ universality class. If the reader is confused by the fact that an apparently minor detail of the growth process (the possibility of adding only one cube at a time or several) may change the qualitative properties of the behaviour on a large scale, it can be reassuring that this is not intuitive even for experts. Indeed, it is not generally easy to guess the universality class of a growth model a priori, just by starting from its definition. On the other hand, it is important to mention that the "anisotropic" model we have just discussed is not an isolated example in the literature. Indeed, there is a whole class of growth models [2] that can be formulated in terms of Schur processes, a combinatorial object introduced by Okounkov and Reshetikhin in relation to plane partitions. The specific model described above is a special case of this class, and results (i)-(iv) have been rigorously demonstrated for several others.

Without going into technical details, it should be pointed out here that a peculiarity of the growth models of [2] (such as the one in Figure s6) which makes them amenable to mathematical analysis is that their stationary measures $\pi_{\rho}$ have the following determinantal property. Given $n$ points $x_{1}, \ldots, x_{n}$ of the plane and $n$ colors $c_{1}, \ldots, c_{n}$, where every $c_{i}$ is one of the three colors of the tiles of a rhombus tiling, let $A=A\left(x_{1}, c_{1} ; \ldots ; x_{n}, c_{n}\right)$ be the event: "there is a rhombus of colour $c_{i}$ at point $x_{i}$, for all $i \leq n$ ". Then, the probability of $A$ is given by the determinant of an $n \times n$ matrix, whose elements are Fourier coefficients of a certain explicit function, defined on the two-dimensional torus $[0,1]^{2}$. This remarkable determinantal property also extends to spatio-temporal correlations in the sense that, under certain restrictions, the probability of events of the type: "there is a rhombus of colour $c_{i}$ at point $x_{i}$ and at time $t_{i}$ for all $i \leq n$ " is also given by a determinant.

In conclusion, it can be said that the mathematical understanding of the growth models of the Anisotropic KPZ class is much more satisfactory than that of the models of the KPZ class as a whole. However, there are two aspects that have remained rather mysterious until very recently:

1. Firstly, for all models of the AKPZ class for which a rigorous study is possible, the proof that $\lambda_{1} \lambda_{2} \leq 0$ requires an explicit and very unenlightening calculation of the second derivatives of $v(\cdot)$, which must be done on a case-by-case basis, and which is based on the explicit formula of $v(\cdot)$. However, according to conjecture 1 formulated above, the negativity of this product should be linked to qualitative properties of the model.

2. In addition, it often happens that although the function $v(\rho)$ has a very complicated expression in terms of the slope $\rho$, it can be rewritten as a very simple harmonic function in terms of a natural complex variable $z(\rho)$, introduced by

Kenyon and Okounkov [8] in the context of dimer models. In a very recent work [3] we shed some light on these two points and we understood that, for all the growth models of the Anisotropic KPZ class known so far, the fact that $\lambda_{1} \lambda_{2} \leq 0$ and the harmonicity property of $v(\cdot)$ have a rather simple geometrical origin. We give here the main steps of our reasoning: 
(1) First of all, one naturally associates a continuous family $\mathcal{F}$ of probability measures $\pi$ with these growth models (this family includes the steady states $\pi_{\rho}$ but also measures that are not invariant by translation). In general, $\pi \in \mathcal{F}$ is not a stationary state of the Markov process. However, if the initial condition is distributed according to $\pi^{(0)} \in \mathcal{F}$, then at any time $t>0$, the law $\pi^{(t)}$ of the interface still belongs to $\mathcal{F}$.

(2) The second observation is that a typical height profile $\{h(x)\}_{x \in \mathbb{Z}^{2}}$, which is randomly drawn according to $\pi \in \mathcal{F}$, is close on a large scale to a minimizer $\phi_{0}$ of a functional of the type

$$
\int_{\mathbb{R}^{2}} \sigma(\nabla \phi) d x
$$

where $\sigma(\cdot)$ is a convex function (surface tension). More explicitly, the fact that $h(\cdot)$ and $\phi_{0}(\cdot)$ are "close on a large scale" means that, with probability 1 ,

$$
\lim _{\epsilon \rightarrow 0} \epsilon h\left(\left\lfloor\epsilon^{-1} x\right\rfloor\right)=\phi_{0}(x) .
$$

The minimizers $\phi_{0}$ of (9) satisfy the associated EulerLagrange equations:

$$
\operatorname{div}\left(\nabla \sigma \circ \nabla \phi_{0}(x)\right)=0,
$$

where div denotes the divergence with respect to spatial variables $x \in \mathbb{R}^{2}$, whereas $\nabla \sigma \circ \nabla \phi_{0}$ denotes the gradient of $\sigma(\rho)$ with respect to its argument $\rho$, computed for $\rho=$ $\nabla \phi_{0}(x)$

(3) From these two facts it follows that, if the initial datum $\phi_{0}$ of the hydrodynamic PDE (4) is a solution to the EulerLagrange equation, this is also the case for the solution $\phi(\cdot, t)$ at any time $t>0$.

(4) This link between the Euler-Lagrange equation (involving $\sigma$ ) and the hydrodynamic equation (involving $v$ ) implies a non-linear relationship between the Hessian $D^{2} v$ of $v(\cdot)$ and the (positive definite) Hessian of $\sigma$. This relation implies in turn that the determinant of $D^{2} v$ is not strictly positive (this last step requires a few lines of computations that we do not develop here).

This is therefore a rigorous first step, no longer based on explicit calculations for a specific model but on general analytical arguments, which sheds some light on conjecture 1. However, the road to its full justification remains very long: one reason is that we barely know anything about the models of the $\mathrm{KPZ}$ class, where $v(\cdot)$ is strictly convex or strictly concave, and about their critical exponents $\alpha, \beta$, which are supposed to be universal.

\section{Bibliography}

[1] A.L. Barabási and H.E. Stanley. Fractal Concepts in Surface Growth. Cambridge University Press, Cambridge, 1995.

[2] A. Borodin and P.L. Ferrari. Anisotropic Growth of Random Surfaces in $2+1$ Dimensions. Comm. Math. Phys., 325:603684, 2014

[3] A. Borodin and F. L. Toninelli. Two-dimensional anisotropic KPZ growth and limit shapes. J. Stat. Mech. Theory Exp. 2018(8):083205, 2018.

[4] P. Caputo, F. Martinelli, and F. L. Toninelli. Mixing times of monotone surfaces and sos interfaces: A mean curvature approach. Comm. Math. Phys., 311(1):157-189, 2012.

[5] I. Corwin. The Kardar-Parisi-Zhang equation and universality class. Random Matrices: Theory Appl., 01:1130001, 2012.
[6] T. Funaki and H. Spohn. Motion by mean curvature from the Ginzburg-Landau $\nabla \phi$ interface model. Comm. Math. Phys., 185:1-36, 1997 .

[7] G. Giacomin, S. Olla, and H. Spohn. Equilibrium fluctuations for $\nabla \varphi$ interface model. Ann. Probab., 29(3): 1138-1172, 2001.

[8] R. Kenyon and A. Okounkov. Limit shapes and the complex Burgers equation. Acta Math., 199:263-302, 2007.

[9] C. Kipnis and C. Landim. Scaling Limits of Interacting Particle Systems. Springer Verlag, Berlin, 1999.

[10] B. Laslier and F. L. Toninelli. Lozenge tilings, Glauber dynamics and macroscopic shape. Comm. Math. Phys., 338(3):1287-1326, 2015.

[11] B. Laslier and F. L. Toninelli. Lozenge tiling dynamics and convergence to the hydrodynamic equation. Comm. Math. Phys., 358(3):1117-1149, 2018.

[12] B. Laslier and F.L. Toninelli. Hydrodynamic limit equation for a lozenge tiling glauber dynamics. Ann. Henri Poincaré, 18(6): 2007-2043, 2017.

[13] H. Spohn. Large Scale Dynamics of Interacting Particles. Texts and Monographs in Physics. Springer Verlag, Heidelberg, 1991.

[14] H. Spohn. Interface motion in models with stochastic dynamics. J. Stat. Phys., 71(5-6):1081-1132, 1993.

[15] Fabio Toninelli. $(2+1)$-dimensional interface dynamics: mixing time, hydrodynamic limit and anisotropic KPZ growth. In Proceedings of the International Congress of Mathematicians - Rio de Janeiro 2018. Vol. III. Invited lectures, pages 2733 2758. World Sci. Publ., Hackensack, NJ, 2018.

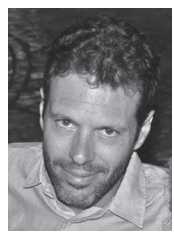

Fabio Toninelli [fabio.toninelli@tuwien.ac.at] is a Professor at TU Wien from February 2020 (on leave from CNRS, Universite Claude Bernard Lyon 1). He obtained his PhD thesis in physics at Scuola normale superiore in Pisa. His main research domains are probability and classical statistical physics.

Acknowledgments. The EMS Newsletter thanks La Gazette des Mathématiciens for authorisation to republish this text, which is an English translation of the paper [La Gazette des Mathématiciens, Number 160, pp. 15-26, April 2019]. 